\title{
Stage IA Vaginal Cancer AJCC v8
}

National Cancer Institute

\section{Source}

National Cancer Institute. Stage IA Vaginal Cancer A/CC v8. NCI Thesaurus. Code C139659.

Stage IA includes: T1a, N0, M0. T1a: Tumor confined to the vagina, measuring $2.0 \mathrm{~cm}$ or less. N0: No regional lymph node metastasis. MO: No distant metastasis. (AJCC 8th Ed.) 